\title{
A Delayed Recrudescent Case of Sigmoidocutaneous Fistula due to Diverticulitis
}

\author{
Takaaki Fujii ${ }^{a, b}$ Toshihiro Nakabayashi ${ }^{a, b}$ \\ Shinji Hashimoto ${ }^{a, b}$ Hiroyuki Kuwano $^{a}$ \\ aDepartment of General Surgical Science, Graduate School of Medicine, Gunma \\ University, and ${ }^{\text {b}}$ Department of Surgery, Gunma Prefectural Cardiovascular \\ Center, Gunma, Japan
}

\section{Key Words}

Colocutaneous fistula $\cdot$ Diverticular disease $\cdot$ Diverticulitis

\begin{abstract}
Colocutaneous fistula caused by diverticulitis is relatively rare, and a delayed recrudescent case of colocutaneous fistula is very uncommon. We herein report a rare case of a Japanese 56-year-old male with delayed recrudescent sigmoidocutaneous fistula due to diverticulitis. A colocutaneous fistula was formed after a drainage operation against a perforation of the sigmoid colon diverticulum. After 5 years from treatment, he was admitted to our hospital because of lower abdominal pain. We diagnosed the recrudescent sigmoidocutaneous fistula by abdominal computed tomography and gastrografin enema, and managed the patient with total parenteral nutrition and antibiotics. As the fistula formation did not improve, a low anterior resection with fistulectomy was performed. The postoperative course was uneventful and the patient was discharged. It has been reported that, in fistulas of the skin caused by diverticular disease, complete closure of the fistula by conservative therapy may not be possible. This case also implies the possibility of a recurrence of the fistula even if the conservative treatment was effective. In cases of colocutaneous fistulas due to diverticulitis, radical surgery is considered necessary because of possibility of recurrence of the fistula.
\end{abstract}

\section{Introduction}

Colonic diverticulosis refers to the formation of small outpouchings from the colonic lumen due to mucosal herniation through the colonic wall at sites of vascular perforation. Although most patients affected will remain entirely asymptomatic, some patients have clinical syndromes, mainly diverticulitis and diverticular haemorrhage [1]. It is also well 
known that diverticular disease of the colon can lead to fistula formation. The commonest fistula of the colon caused by diverticular disease is a colovesical fistula, and colocutaneous fistula is relatively rare [2-4]. Furthermore, a delayed recrudescent case of colocutaneous fistula is very uncommon. We herein report a rare case of delayed recrudescent sigmoidocutaneous fistula due to diverticulitis. In this case, conservative therapy was not effective and low anterior resection with fistulectomy was performed. This case also implies the possibility of recurrence of the fistula even if the conservative treatment was effective.

\section{Case Report}

On 12 February 2007, a 56-year-old Japanese man was admitted to our hospital with a complaint of lower abdominal pain. The patient had been suffering from colocutaneous fistula after a drainage operation against perforation of the sigmoid colon diverticulum. A drainage procedure and a closure of the perforated colon with covering stoma were performed at that time. After 5 years of symptom-free period, he was admitted to our hospital because of lower abdominal pain around the scar of the surgical wound left after the drainage. This area featured redness, induration, and tenderness.

On admission, a physical examination revealed that the patient had a height of $169 \mathrm{~cm}$, a weight of $62.5 \mathrm{~kg}$, a heart rate of 92 beats per minute with regularity, and blood pressure of $125 / 91 \mathrm{~mm} \mathrm{Hg}$. His white blood cell count was $11,670 / \mathrm{mm}^{3}$, and C-reactive protein was $10.08 \mathrm{mg} / \mathrm{dl}$. Because of the inflammatory reaction, the incision and drainage procedures were performed and the patient was treated with antibiotics. Abdominal radiography revealed no remarkable findings. Computed tomography of the abdomen showed a high-density area around the site of drain insertion and the rectus abdominis muscle in contact with the diverticulum of the sigmoid colon (fig. 1a, b). From the computed tomography findings, sigmoidocutaneous fistula due to diverticulitis was clinically suspected, and we diagnosed a recrudescent sigmoidocutaneous fistula by gastrografin enema (fig. 2). This enema revealed diverticulosis of the sigmoid colon and fistula formation of the sigmoid colon near the rectum. The diagnosis was confirmed by fecal exudates observed from cutaneous fistula in the course of conservative treatment. A diagnosis of Crohn's disease was denied by colonscopic appearance. The patient was managed with total parenteral nutrition and antibiotics. The abdominal pain improved after treatment began, but the fistula formation did not improve, as confirmed by gastrografin enema. As conservative treatment was not effective, an operation was performed. During surgery it was found that apparent adhesion and fibrosis were located mainly around the rectum to the sigmoid colon, where the perforated diverticulum of the sigmoid colon was recognized. This inflammatory and fibrosing process partly involved ileum. The fistula wall consisted partly of serosa of the ileum and bladder wall, and the fistula was continuous with cutaneous tissue. Granulomatous inflammation and fibrosis were also observed at the fistula wall, composed of ileum or bladder. From these findings, sigmoidocutaneous fistula due to diverticulitis was diagnosed and a low anterior resection with fistulectomy was performed (fig. 3a, b). The postoperative course was uneventful and the patient was discharged.

\section{Discussion}

We here report a rare case of delayed recrudescent sigmoidocutaneous fistula due to diverticulitis. Fistulas are a relatively common complication of diverticular disease of the sigmoid colon [5]. Fistulas with diverticular diseases may involve any pelvic structure including urinary bladder, vagina, uterus, fallopian tube, ureter, other parts of the colon or ileum, and cutaneous tissue. The colovesical fistula is the most common internal fistula, and a colocutaneous fistula is relatively uncommon [2-4]. A previous study reported that the type of fistulas included colovesical (48\%), colovaginal (44\%), coloenteric (2\%), colotubal (2\%), and colocutaneous (4\%) fistulas [3]. Colocutaneous fistula occurs almost exclusively as a complication of operation, and spontaneous colocutaneous fistulas are rare $[5,6]$. The cause for the fistula fomation of this case was also considered attributable to the previous operation. Interestingly, this case was a recurrent case of fistula. Clinically, with conservative therapy it may be difficult to cure 
the fistula completely. Surgery is usually undertaken for fistulas complicating diverticular disease of the colon. Thus, recurrent cases of fistula of the colon caused by diverticular disease are very uncommon. Furthermore, this case implies that radical surgery is needed to remove a fistula due to diverticular disease, because this case shows the possibility of a recurrence of the fistula even though the conservative treatment was effective.

A complicating disease with diverticulitis, such as an abscess or phlegmon, can be managed conservatively, and subsequent surgery is selective depending on the recovery from the initial episode. However, a fistula often requires surgery [7]. Certainly, some reports have shown that a fistula can be managed with a course of nonoperative treatment $[3,8]$. However, most fistulas due to diverticulitis are dealt with by a surgical procedure and can be safely managed by resection and primary anastomosis in many cases $[2-4,9$, 10]. Conservative therapy may help improve acute inflammatory reaction and may make operations easier, but closure of the fistula by conservative treatment may not be possible. Clinically, a patient with a fistula should first be managed with total parenteral nutrition and antibiotics to reduce inflammation, and only then should an operation be performed. In this case, after conservative therapy, a low anterior resection with fistulectomy was performed and provided good results.

In conclusion, we here reported a rare case of delayed recrudescent sigmoidocutaneous fistula due to diverticulitis. In cases of colocutaneous fistulas due to diverticulitis, radical surgery should be considered necessary because of possibility of a recurrence of the fistula even though the conservative treatment was effective. 
Fig. 1. a, b Computed tomography of the abdomen showed a high-density area around the site of drain insertion and the rectus abdominis muscle in contact with the diverticulum of the sigmoid colon.
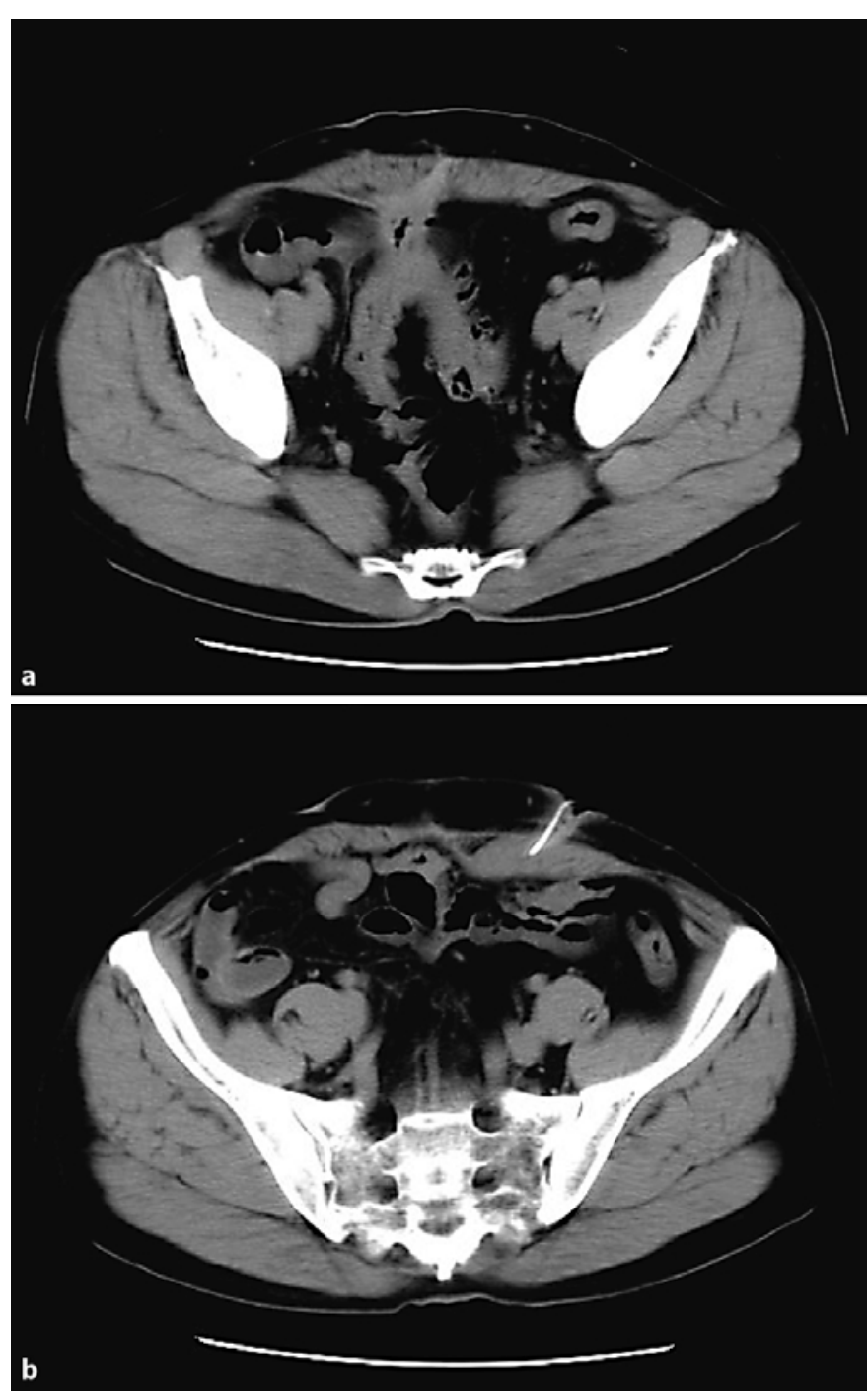
CaseReportsin |Case Rep Gastroenterol 2007;1:116-122 Gastroenterology

Fig. 2. Gastrografin enema showed diverticulosis of the sigmoid colon and fistula formation of the sigmoid colon near the rectum.

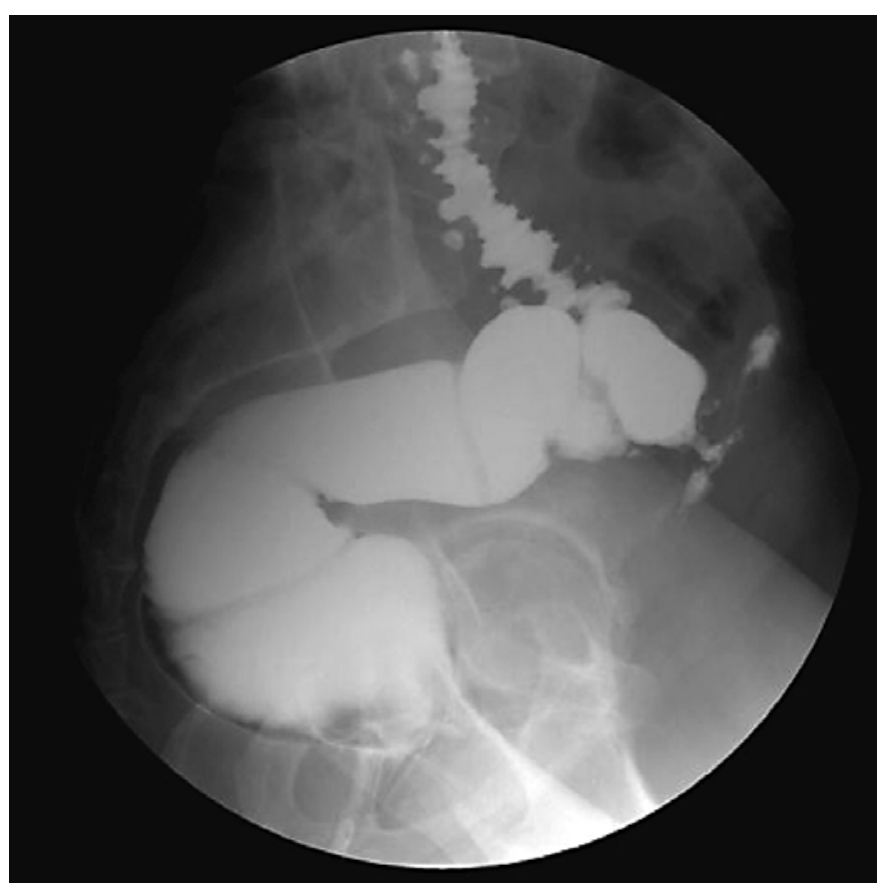


Fig. 3. a, b Resected specimen showed a perforated diverticulum of the sigmoid colon, where the fistula was recognized. The fistula was continuous with the skin.
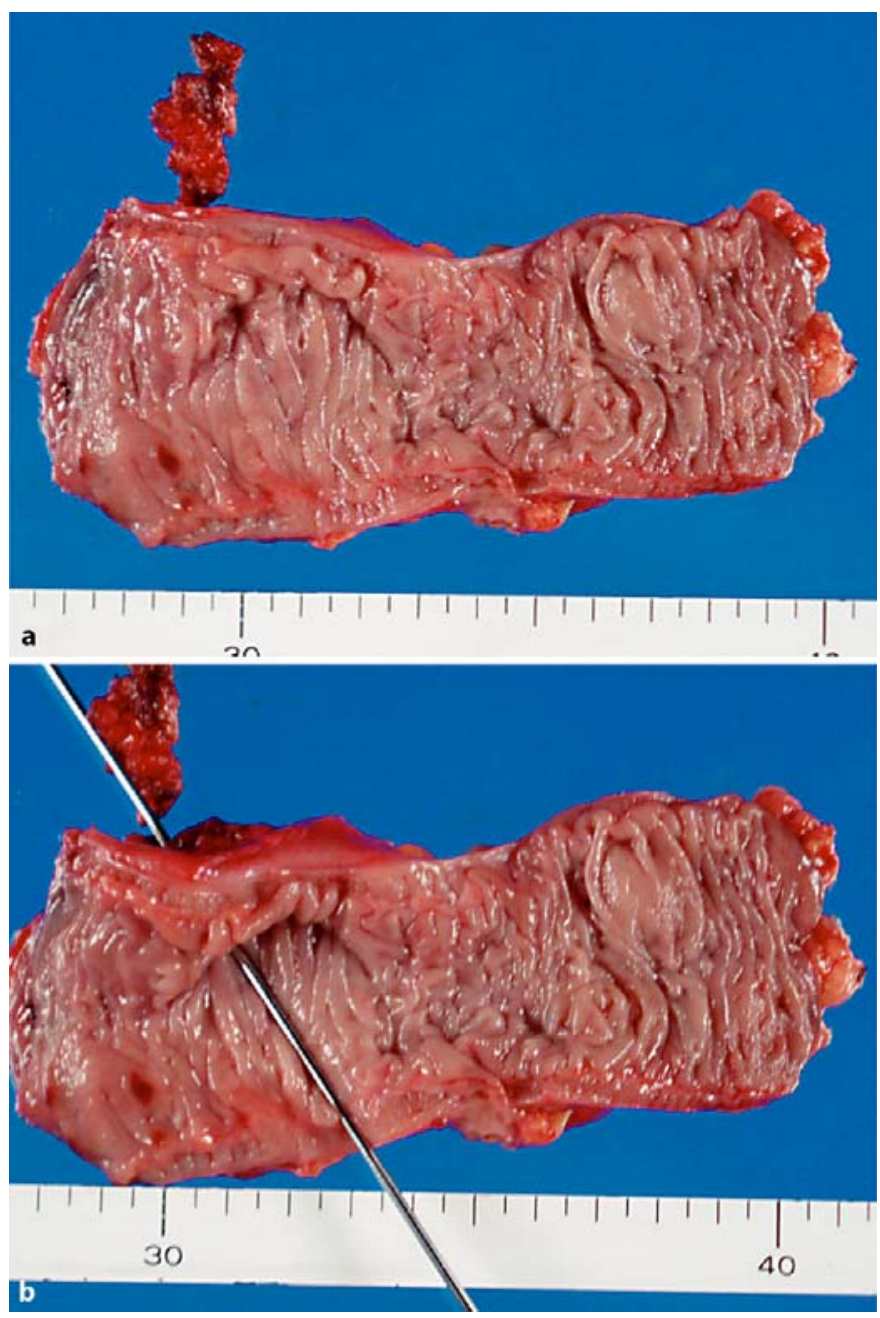


\section{References}

1 Stollman N, Raskin JB: Diverticular disease of the colon. Lancet 2004;363:631639.

2 Rodkey GV, Welch CE: Changing patterns in the surgical treatment of diverticular disease. Ann Surg 1984;200:466-478.

3 Vasilevsky CA, Belliveau P, Trudel JL, Stein BL, Gordon PH: Fistulas complicating diverticulitis. Int J Colorectal Dis 1998;13:57-60.

4 Bahadursingh AM, Virgo KS, Kaminski DL, Longo WE: Spectrum of disease and outcome of complicated diverticular disease. Am J Surg 2003;186:696-701.

5 Colocok BP, Stahmann FD: Fistulas complicating diverticular disease of the sigmoid colon. Ann Surg 1972;175:838-846.

6 Rao UP, Venkitachalam PS, Posner GL, Estuita ET: Diverticulitis manifesting as transverse colocutaneous fistula: report of a case and review of literature. Dis Colon Rectum 1980;23:44-48.

7 Frattini J, Longo WE: Diagnosis and treatment of chronic and recurrent diverticulitis. J Clin Gastroenterol 2006;40:S145-S149.

8 Amin M, Nallinger R, Polk HC Jr: Conservative treatment of selected patients with colovesical fistula due to diverticulitis. Surg Gynecol Obstet 1984;19:442444.

9 Pontari MA, McMillen MA, Garvey RH, Ballantyne GH: Diagnosis and treatment of enterovesical fistulae. Am Surg 1992;58:258-263.

10 Wedell J, Banzhaf G, Chaoui R, Fischer R, Reichmann J: Surgical treatment of complicated colonic diverticulitis. Br J Surg 1997;84:380-383. 